\title{
PHOSPHOGLYCOLIPID PROFILING OF BACTERIAL ENDOTOXINS
}

\author{
ANIKó KILÁR ${ }^{* 1,2}$, ÁGNES DÖRNYEI ${ }^{2}$, VIKTOR SÁNDOR ${ }^{1}$, FERENC KILÁR ${ }^{1}$, AND BÉLA KOCSIS ${ }^{3}$ \\ ${ }^{1}$ Institute of Bioanalysis, University of Pécs, Szigeti út 12, Pécs, 7624, HUNGARY \\ ${ }^{2}$ Department of Analytical and Environmental Chemistry and Szentágothai Research Centre, University of \\ Pécs, Ifjúság útja 6, Pécs, 7624, HUNGARY \\ ${ }^{3}$ Department of Medical Microbiology and Immunology, University of Pécs, Szigeti út 12, Pécs, 7624, \\ HUNGARY
}

\begin{abstract}
Much interest is at present focused on bacterial endotoxins, also known as lipopolysaccharides (LPS), as they are responsible for the development of clinical symptoms of Gram-negative sepsis which is the leading cause of death in intensive care units. Endotoxicity is associated with the special phosphoglycolipid part of LPS, termed lipid A. Main challenges in the structural elucidation of lipid A arise from its amphiphilic character and inherent heterogeneity. A mass spectrometrybased de novo method combined with reversed-phase liquid chromatography for the detailed structural characterization of complex lipid A mixtures (obtained by mild acid hydrolysis of LPS) from different bacterial sources has been developed. Tandem mass spectrometric measurements were performed with an electrospray-ionisation quadrupole time-of-flight (ESI-Q-TOF) mass spectrometer in both negative- and positive-ionization modes in order to explore fragmentation pathways. It was found that characteristic product ions in the positive-ion mode could be used for the unambiguous assignment of the phosphorylation site, whereas the use of both ionization modes provided consistent and/or complementary information about the fatty acyl distribution between the two glucosamine moieties of lipid A. Since the immunostimulatory (advantageous) vs. proinflammatory (endotoxic) effect of the lipid A is closely related to the fine chemical structure, our relatively simple structural elucidation strategy could offer great potential in the bioanalysis of native lipid $A$ samples and lipid A-based vaccines.
\end{abstract}

Keywords: lipid A, HPLC, tandem mass spectrometry, positive-ion mode, negative-ion mode

\section{Introduction}

Bacterial endotoxins are important initiators of clinical symptoms of Gram-negative sepsis, a serious medical condition with a high mortality rate among patients in intensive care units worldwide. Endotoxins, chemically lipopolysaccharides (LPS) or lipooligosaccharides (LOS), are found on the surface of Gram-negative bacteria. They are essentially composed of two regions: a heteropolysaccharide chain (divided into the oligosaccharide core and the O-side chain - this component is missing from LOS) that extends outwards from the bacterial cell surface, and a phosphoglycolipid, termed lipid A, which is anchored in the outer membrane (Fig. 1). Specifically, the endotoxic activity is related only to the lipidic domain of endotoxins.

With regard to the toxicity of lipid A the crucial factors are the acylation and phosphorylation status. This most biologically potent molecule, capable of inducing septic shock, consists of an asymmetrically hexaacylated, bisphosphorylated glucosamine disaccharide [1]. Other lipid A species that deviate from this structure have lower endotoxicity or may even have beneficial

\footnotetext{
*Correspondence: aniko.kilar@aok.pte.hu
}

effects. Consequently, the structural analysis of the lipid A moiety is of fundamental interest in terms of clinical aspects and vaccine development.

The structural characterization of lipid A poses a unique analytical challenge, as lipid A isolated from a single bacterial strain generally consists of a mixture of differently acylated and phosphorylated species. Furthermore, the relative abundances of the different structures can vary with growth conditions, e.g. temperature, culture

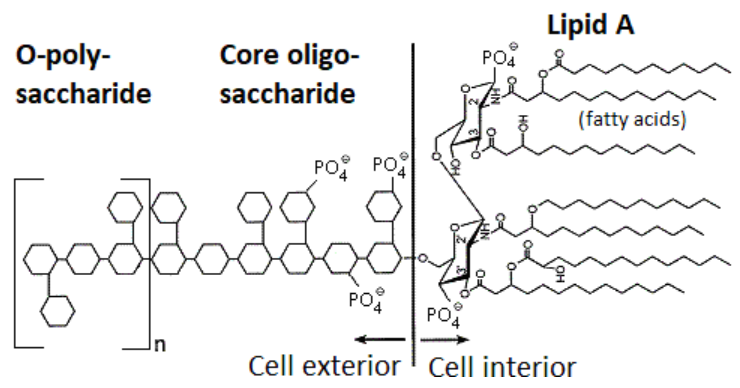

Figure 1: Schematic representation of the chemical structure of an enterobacterial outer membrane lipopolysaccharide (endotoxin). 
media and $\mathrm{pH}$. Within this field, tandem mass spectrometry (MS/MS or MSn) has emerged as a core technology for the in-depth structural elucidation of lipid A species [2]. However, separation and simultaneous quantification of singular components by powerful separation methods prior to MS would be highly desirable to adequately characterize the heterogenic structures.

Here, a brief presentation of an alternative method to multiple-stage MS for the differentiation of various lipid A species - including isobars - within a bacterial sample is provided. Our method is based on a reversedphase high-performance liquid chromatography (RPHPLC) separation and online ESI-Q-TOF mass spectrometric detection of molecular species in native lipid A isolates. The exact structures of the lipid A molecules in the complex mixtures were deduced from specific fragmentation patterns obtained in both the negative- $[3,4]$ and positive-ion modes [5].

\section{Experimental}

\subsection{Chemicals and Reagents}

Methanol, isopropyl alcohol, water (LC-MS grade), dichloromethane (HPLC, $\geq 99.9 \%)$, triethylamine $\left(\mathrm{Et}_{3} \mathrm{~N}\right)$ and acetic acid $(\mathrm{AcOH})$ were purchased from SigmaAldrich (Steinheim, Germany).

\subsection{Bacterial strains and lipid A isolation}

The strains Escherichia coli $\mathrm{O} 111$ and Proteus morganii O34 were cultured in a laboratory fermenter, and then collected by centrifugation. The bacterial LPS was extracted from acetone-dried organisms by the traditional hot phenol-water procedure and lyophilized.

Lipid A was released from LPS by mild-acid hydrolysis with $1 \%(\mathrm{v} / \mathrm{v}) \mathrm{AcOH}$ at $100^{\circ} \mathrm{C}$ for $1 \mathrm{~h}$, then the solution was centrifuged $\left(8000 \times \mathrm{g}, 4^{\circ} \mathrm{C}, 20 \mathrm{mins}\right)$. The sediment (containing lipid A) was washed four times with distilled water and lyophilized. Lipid A samples were dissolved in methanol/dichloromethane $(95: 5, \mathrm{v} / \mathrm{v})$ at a final concentration of $0.5 \mathrm{mg} / \mathrm{ml}$.

\subsection{Liquid Chromatography - Mass Spec- trometry Measurements}

Separation and mass spectrometric detection of lipid A samples were performed with an 1290 Infinity Ultra-high performance liquid chromatography (UHPLC) system (Agilent Technologies, Waldbronn, Germany) coupled to a 6530 Accurate-Mass Q-TOF LC/MS system (Agilent Technologies, Singapore), using a Kinetex ${ }^{\mathrm{TM}}$ coreshell C18 column $(100 \mathrm{~mm} \times 4.6 \mathrm{~mm}, 2.6 \mu \mathrm{m}$ particle size, Phenomenex). Mobile phase A was methanol/water (95:5, v/v) and mobile phase B was isopropyl alcohol, both containing $0.1 \% \mathrm{Et}_{3} \mathrm{~N}$ and $0.1 \% \mathrm{AcOH}(\mathrm{v} / \mathrm{v})$. The injection volume was $3 \mu \mathrm{l}$. Separation was performed at

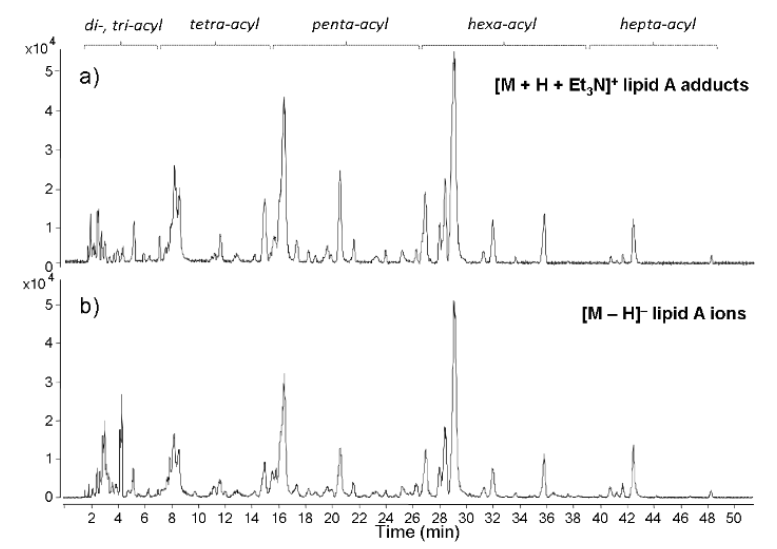

Figure 2: HPLC-MS base peak chromatograms of the native, heterogeneous E. coli $\mathrm{O} 111$ lipid A preparation acquired in the a) positive- and b) negative-ion modes.

a constant flow rate of $0.5 \mathrm{ml} / \mathrm{min}$ at $50^{\circ} \mathrm{C}$. A linear gradient of 50 min started at $0 \%$ of mobile phase B and ramped up to $50 \%$ of mobile phase B, and was then maintained at $50 \%$ of mobile phase B for 2 mins.

Negative- and positive-ion mass spectra of the column eluate were recorded between $\mathrm{m} / \mathrm{z}, 100$ and 3,200. The ESI ion source was operated using the following conditions: pressure of nebulizing gas $\left(\mathrm{N}_{2}\right), 30 \mathrm{psi}$; temperature and flow rate of drying gas $\left(\mathrm{N}_{2}\right), 325^{\circ} \mathrm{C}$ and $8 \mathrm{l} / \mathrm{min}$, respectively; temperature and flow rate of sheath gas, $300^{\circ} \mathrm{C}$ and $11 \mathrm{l} / \mathrm{min}$, respectively; capillary voltage, $4 \mathrm{kV}$; nozzle voltage, $2 \mathrm{kV}$; fragmentor potential, $100 \mathrm{~V}$; and skimmer potential, $70 \mathrm{~V}$. For the targeted MS/MS analysis, the following parameters were used: mass range, $m / z$, 70 2000; acquisition rate, $333.3 \mathrm{~ms} / \mathrm{scan}$; quadrupole isolation width, $1.3 \mathrm{~m} / \mathrm{z}$; collision energy, 70-100 V; maximum number of precursor ions/cycle, 4 ; and active charge state of the precursor ion, 1.

\section{Results and Analysis}

\subsection{HPLC-MS of bacterial lipid A}

Crude lipid A preparations from E. coli $\mathrm{O} 111$ and $P$. morganii $\mathrm{O} 34$ bacteria were used as training sets for the development of the method. The HPLC separation conditions were optimized in order to find a balance between solubility, adequate separation and ionization of the intact lipid A's. Fig. 2 shows the HPLC-MS analysis in the positive- and negative-ion modes for the E. coli O111 lipid A isolate, only. It was found that the lipid A samples expressed extreme structural heterogeneity, showing the presence of numerous bis-, mono- and nonphosphorylated structures and diverse acylation states ranging from di- to hepta-acylation. The most abundant chromatographic peak from both bacteria was a hexaacylated, monophosphorylated species. The elution order of the separated compounds was consistent with their relative hydrophobicity, defined mainly by the total num- 
ber and length of hydrophobic acyl chains, followed by the number of polar phosphate groups. Several isomers of identical mass could be identified in which the length and/or position of the acyl chains varied.

Since the phosphoglycolipids are acidic in nature, they could readily be ionized as deprotonated ions, i.e. $[\mathrm{M}-\mathrm{H}]^{-}$in the negative-ion mode, whereas their ionization in the positive-ion mode was enhanced by adduct formation with triethylamine used as an eluent additive, i.e. $\left[\mathrm{M}+\mathrm{H}+\mathrm{Et}_{3} \mathrm{~N}\right]^{+}$adducts were formed.

\subsection{Tandem mass spectrometric analysis of lipid A species}

In order to investigate the fragmentation behavior under low-energy collision-induced dissociation (CID) conditions of each separated lipid A precursor ion, ESIMS/MS experiments were performed in both ionization modes. Based on previously published data on the acyl distribution of the C4'-monophosphorylated, hexaacylated lipid A molecule from E. coli O111 [6], ions observed in the tandem mass spectra (not shown) of this lipid A species (appearing at $t_{\mathrm{R}}=29.2 \mathrm{mins}$ in Fig. 2) could be structurally assigned in both ionization modes $[3,5]$. Using this as a standard, the CID mass spectral profiles of other monophosphorylated lipid A species separated by chromatography were compared, and the fragmentation preferences for each of them, in both ionization modes, were explored. Fig. 3 presents an example of the correlation between the phosphoglycolipid structure and the fragmentation profiles in the case of the main hepta-acylated lipid A compound $\left(t_{\mathrm{R}}=42.5 \mathrm{mins}\right.$ in Fig. 2) from $E$. coli O111. The site-specific cleavages are assigned with a number denoting the carbon of the glucosamine to which the substitution is attached, and a Greek letter designating the position of the bond which was cleaved.

In the tandem mass spectrum of the $\left[\mathrm{M}+\mathrm{H}+\mathrm{Et}_{3} \mathrm{~N}\right]+$ triethylammonium adduct of this lipid A species (Fig. 3a), only a few ions resulting from the neutral loss of acyl chains are present, however, in that of the deprotonated form (Fig. 3b), the abundances of product ions in the higher $\mathrm{m} / \mathrm{z}$ region straightforwardly indicated the consecutive dissociation order of the ester-linked fatty acids. Thus, their position could be assigned. Each tandem mass spectrum also exhibited diagnostic intra-ring (A-type ions in the negative-ion mode) or inter-ring (Band Y-type ions in the positive-ion mode) cleavage products, which confirmed the substitution position of the fatty acids on the two glucosamine residues.

Fig. 4 shows the tandem mass spectra of the $\mathrm{C} 1$ phosphorylation isomer of this hepta-acylated lipid A species. The location of the single phosphoryl group (i.e. at $\mathrm{C} 1$ or C4') could be directly assessed from the positive-ion mode tandem mass spectra by calculating the mass difference between the $m / z$ of the precursor ion and that of the product ion resulting from the cleavage of the $1 \alpha$ bond
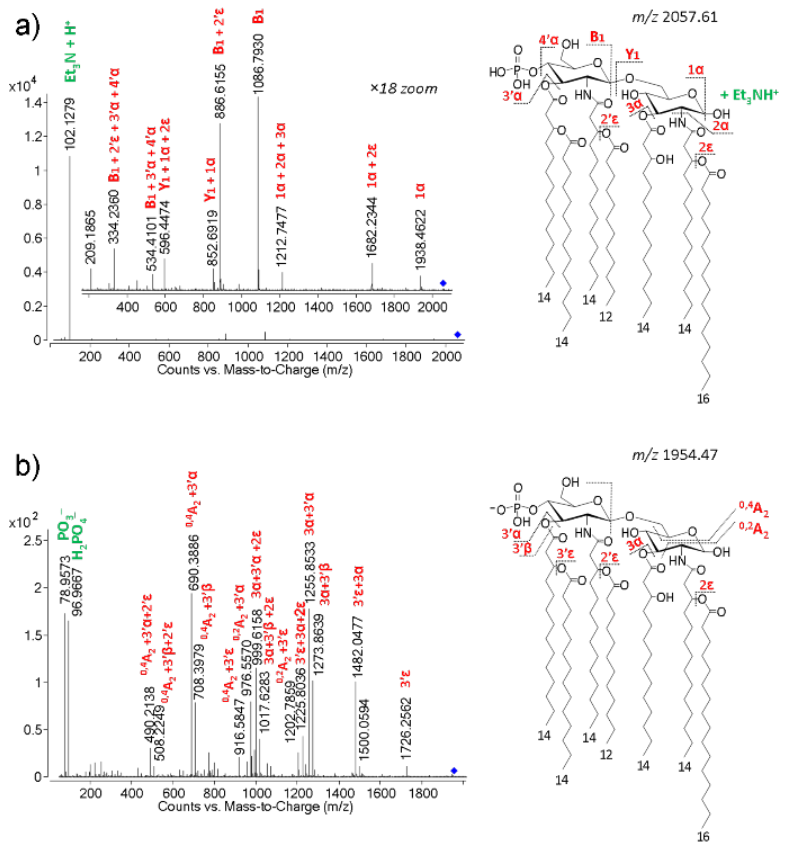

Figure 3: Tandem mass spectra in a) positive- and b) negative-ion modes and a depiction of the identified structure of the C4'-monophosphorylated hepta-acylated lipid A from the E. coli $\mathrm{O} 111$ bacterium. Site-specific cleavages are indicated next to the ion signals.

(compare Figs. 3a and 4a). Namely, the mass difference of $199 \mathrm{u}$ corresponded to the combined loss of triethylamine and an orthophosphoric acid molecule, indicating that a phosphoric acid molecule had esterified the $\mathrm{C} 1$ hydroxyl group of the reducing end. On the other hand, a mass difference of $119 \mathrm{u}$ resulted from the combined loss of triethylamine and a water molecule, indicating the presence of a free hydroxyl group at $\mathrm{C} 1$ (consequently, the phosphoryl group must be located at $\mathrm{C}^{\prime}$ ').

The change in the phosphorylation site also resulted in a distinctive tandem mass spectrum in the negative- ion mode (Fig. 4b). The main differences were the complete lack of A-type cross-ring fragments, and instead the appearance of Z-type inter-ring cleavage products. Furthermore, the intensities of the product ions resulting from the neutral loss of acyl chains were of about the same magnitude, indicating strong competition between the fatty acyl eliminations during the CID process, instead of their stepwise dissociation (compare with Fig. 3b).

The fragmentation behavior of non- and bisphosphorylated lipid A species under both negative and positive CID conditions was investigated, as well. For these, a different sequencing of the acyl-chain cleavages could be observed in the negative-ion mode [3,4], whereas similar fragmentation pathways to that of the monophosphorylated species in the positive-ion mode were identified [5]. Besides the A-type ions (nonreducing end), complementary X-type fragment ions (reducing end) appeared in the negative-ion mode tandem mass 

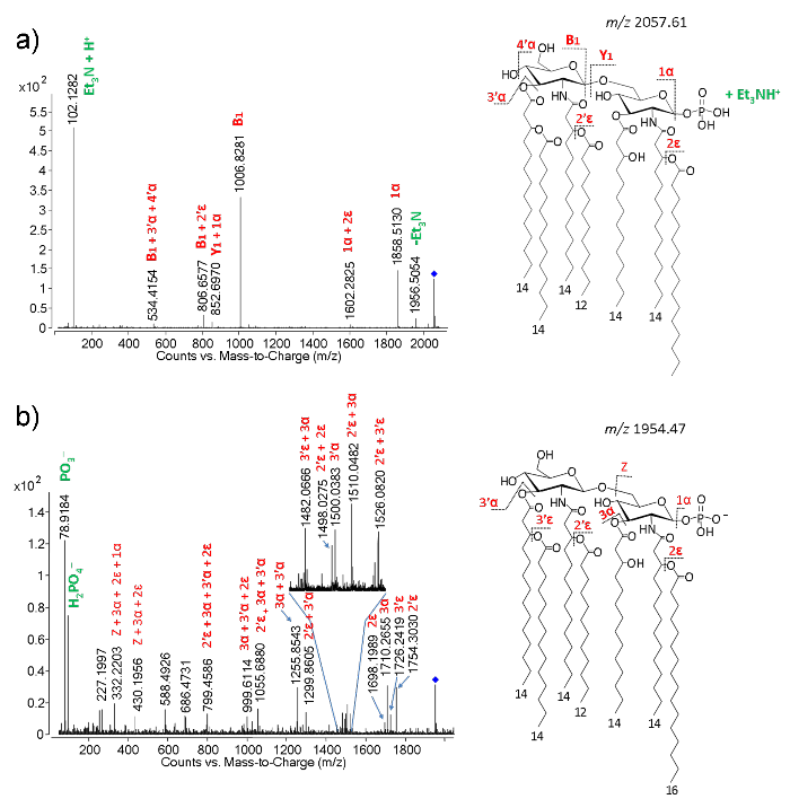

Figure 4: Tandem mass spectra in the a) positive- and b) negative-ion modes and depiction of the identified structure of the C1-monophosphorylated hepta-acylated lipid A from E. coli $\mathrm{O} 111$.

spectra of the nonphosphorylated lipid A species [4].

\section{Discussion}

The online HPLC-MS/MS methodology enabled the simultaneous structural characterization of both phosphorylated and nonphosphorylated lipid A variants within a single run. The main advantage of using the collision cell of a Q-TOF mass spectrometer was that several generations of precursor/product ions - that would otherwise be generated only at higher MS stages with ion-trap experiments - were observed simultaneously in the tandem mass spectra of the separated lipid A species. This facilitated new fragmentation rules to be set for the variety of phosphorylated and acylated lipid A compounds by a simple MS/MS experiment [3-5]. Specifically, the acylation profile of the non-, C4'-mono and bisphosphorylated lipid A species could be inferred partly from the positiveand fully from the negative-ion mode MS/MS analyses [3-5], whereas the complementary use of both ionization modes $[3,5]$ was needed for the full structural characterization of C1-monophosphorylated lipid A.

As an example, the complete structural elucidation strategy by MS/MS of a hepta-acylated C1'monophosphorylated lipid A is as follows. First, observation of the $1 \alpha$ cleavage product in the positive-ion mode is essential to clarify the site of phosphorylation (vide supra). Next, the linkages at the $2^{\prime} \varepsilon$ (only from the Btype ion) and $2 \varepsilon$ positions can be determined from the positive-ion mode analysis. By knowing the fragmentation preference of the secondary ester bonds (which is $2^{\prime} \varepsilon>3^{\prime} \varepsilon>2 \varepsilon[5]$ ), substitutions at these sites can be deconvoluted from the negative-ion mode analysis by observing the $2^{\prime} \varepsilon, 3^{\prime} \varepsilon, 2 \varepsilon$, and $3 \alpha$ cleavage products in the upper $m / z$ region and those resulting from the $3^{\prime} \alpha$, $2^{\prime} \varepsilon+3^{\prime} \varepsilon, 2^{\prime} \varepsilon+2 \varepsilon, 2^{\prime} \varepsilon+3 \alpha$, and $3^{\prime} \varepsilon+3 \alpha$ cleavages displayed in the middle $m / z$ region. Here, the dephosphorylated Z-type ion in the lower $\mathrm{m} / \mathrm{z}$ region indirectly specifies the linkage at the $2 \alpha$ position. Finally, the remaining 2' $\alpha$ substitution can be deduced from the intact B-type ion (positive-ion mode).

\section{Conclusion}

An efficient HPLC separation before the MS detection of natural lipid A mixtures has proven particularly useful in terms of revealing the diversity and relative amount of the various lipid A structures present in bacteria. Such investigations could help to explore the relative contributions of different lipid A structures to the overall activation of the innate immune system. Moreover, knowledge of the chemical and biological aspects of lipid A heterogeneity is crucial in order to design antimicrobial drugs which overcome the evasion strategy of the pathogen. Our technique could be well suited for phosphoglycolipid profiling from different bacterial strains or vaccine preparations.

\section{Acknowledgement}

This research was supported by the grants OTKA K125275 and ÚNKP-17-4-III provided by the New National Excellence Program of the Ministry of Human Capacities.

\section{REFERENCES}

[1] Munford, R.S.: Sensing gram-negative bacterial lipopolysaccharides: a human disease determinant? Infect. Immun. 2008, 76(2), 454-465. DOI: 10.1128/IAI.00939-07

[2] Kilár, A.; Dörnyei, Á.; Kocsis, B.: Structural characterization of bacterial lipopolysaccharides with mass spectrometry and on- and off-line separation techniques. Mass Spectrom. Rev. 2013, 32(2), 90-117. DOI: 10.1002/mas.21352

[3] Sándor, V.; Dörnyei, Á.; Makszin, L.; Kilár, F.; Péterfi, Z.; Kocsis, B.; Kilár, A.: Characterization of complex, heterogeneous lipid A samples using HPLC-MS/MS technique I. Overall analysis with respect to acylation, phosphorylation and isobaric distribution. J. Mass Spectrom. 2016, 51(11), $1043-$ 1063. DOI: 10.1002/jms.3839

[4] Sándor, V.; Kilár, A.; Kilár, F.; Kocsis, B.; Dörnyei, Á.: Characterization of complex, heterogeneous lipid A using HPLC-MS/MS technique II. Structural elucidation of non-phosphorylated lipid A by negativeion mode tandem mass spectrometry. J. Mass Spectrom. 2016, 51(8), 615-628. DOI: 10.1002/jms.3786 
[5] Sándor, V.; Kilár, A.; Kilár, F.; Kocsis, B.; Dörnyei, Á.: Characterization of complex, heterogeneous lipid A samples using HPLC-MS/MS technique III. Positive-ion mode tandem mass spectrometry to reveal phosphorylation and acylation patterns of lipid A. J. Mass Spectrom. 2018, 53(2), 146-161. DOI: $10.1002 / \mathrm{jms} .4046$
[6] Madalinski, G.; Fournier, F.; Wind, F.L.; Afonso, C.; Tabet, J.C.: Gram-negative bacterial lipid A analysis by negative electrospray ion trap mass spectrometry: stepwise dissociations of deprotonated species under low energy CID conditions. Int. J. Mass Spectrom. 2006, 249-250, 77-92. DOI: 10.1016/j.ijms.2005.12.049 